\title{
ADHD in the Early Years: Pre-Natal and Early Causes and Alternative Ways of Dealing
}

\author{
https://doi.org/10.3991/ijoe.v15i13.11203
}

\author{
Anna-Maria Driga $\left.{ }^{\bowtie}\right)$, Athanasios Drigas \\ N.C.S.R. "Demokritos", Athens, Greece \\ anna.maria.driga@gmail.com
}

\begin{abstract}
Attention Deficit Hyperactivity Disorder (ADHD) is a complex neurological disorder with a lack of an official scientific understanding of its genetic nature and the potential causes. However, there are practical research data that points to certain directions. In this paper, we examine the possible external causes of the disorder and specifically ones whose influence is related to pregnancy and (early) childhood years. Children born prematurely and/or with low birth weight are at high risk of developing ADHD. Maternal smoking, alcohol consumption and psychological disorders during pregnancy were proven to play a part as well. Some special social and economic situations raised the risk of appearance in the minors experiencing them. Electronics are a factor of distraction to children and were found to exacerbate symptoms. Most importantly, parenting choices can be determinant for a child with the disorder. A solution often proposed for symptoms of this nature is medication. Nonetheless, there were many defects and risks found within the psychostimulants used for the disorder. Alternative treatments are proposed before reaching for medication, two very effective ones being brain training and meditation.
\end{abstract}

Keywords-ADHD, ADHD Causes, External ADHD Causes, Alternative ADHD Treatments

\section{Introduction}

\subsection{What is ADHD? How does it work?}

Attention deficit hyperactivity disorder (ADHD) is a neurological disorder, characterized by lack of attention and impulsivity. The former is a result of a dysfunction in areas of the brain that control certain abilities, known as executive function. Executive skills consist of short-term memory, flexible thinking and self-regulation. Those skills set the foundation for a person's attention, organization abilities, focusing on assignments, emotion control and self-assessment. It is one of the most common neurological disorders of the generation, especially in children. Despite that fact, so far there has been insufficient research around the topic and thus leaving gaps in the general understanding of its mechanisms. In essence, ADHD is a very complex disorder not yet fully understood. [1] [2] 


\section{Genetic Background and External Factors}

According to existing research ADHD usually runs in families. Although, the exact genetic correlations are yet to be determined. It has been found that children of parents with ADHD have more than $50 \%$ chance of having it themselves. If a child in a family has been diagnosed with ADHD, other children and future children of the family have a $30 \%$ plus chance of having it. [3]

The genetic basis of the disorder is the main foundation for its occurrence. Apart from that, there are further developmental, environmental and psychological factors that can build the condition up and magnify it. ADHD can be a result of the combination of genetic factors with certain environmental influences. Many of the environmental factors can be controlled and in this way help significantly curb the intensity of the disorder. Furthermore, overcoming certain psychological obstacles can be liberating for a patient of ADHD to experience a better life.

\subsection{Gaps and complexity}

ADHD is a very complex disorder and its complexity is widely recognized. Due to the fact that genetic connections are yet to be specified, the range of their impact remains unknown. This also results in the uncertainty of the impact that external factors possess. The evidence for environmental factors is solely based on practical research data, without yet an unshakeable theoretical scientific conclusion.

\section{Prenatal Factors}

\subsection{Premature / Preterm birth}

For many years there have been talks about a link in preterm birth and ADHD symptoms. Many studies have been conducted, however, the sample remains inadequate. Even so, when preterm-born brains are compared with normal ones, there are evident differences. S.N James et al concluded through thorough brain examination of adolescent brains from both categories that preterm-born brains do indeed lack in function in areas associated with ADHD. In addition to that, it was found that premature born individuals have further neurological and cognitive risks. [4]

\subsection{Low birth weight}

Another subject that is often interlinked with premature birth is low birth weight. According to a meta-analysis comparing results and data from multiple studies, low birth weight can be associated with ADHD. The data comparisons showed that the lower the birth weight of an individual, the higher the risk of ADHD appearance. There are three groups of newborns with low-birth-weight according to the severity of weight deficiency. In specific, the first group, extremely premature/extremely low birth weight individuals, have 4 times higher ADHD risk than ones born with normal 
weight. The second group, very premature/very low birth weight presents 2 times higher risk. In those cases, it is advised to get expert help as early as possible to have the ability to gain control of the symptoms. [5]

\subsection{Smoking during pregnancy}

It is well known that fetal tobacco exposure reserves many health and cerebral risks for the child. Those risks include parts of the brain connected to Attention DeficitHyperactivity Disorder. A wide range of studies regarding maternal smoking and the disorder have been lead. Most of those studies appear to verify the link between the two. [6] It is worth noting that newer research suggests that paternal smoking and the mother being exposed to tobacco before birth are just as associated with ADHD as maternal smoking itself. This may unveil a potential gap in the understanding of the said association and possibly unmeasured factors in the function. [7]

Given the numerous uncontrolled causes of ADHD, this hazardous habit should be avoided at all costs during pregnancy.

\subsection{Alcohol consumption in pregnancy}

Much like smoking during pregnancy, maternal alcohol consumption is associated with many health risks and behavioral disorders. More often than not, children with fetal alcohol syndrome are also diagnosed with ADHD. Those children often present ADHD-like symptoms of hyperactivity, impulsivity, unruliness, inattention and slow processing. It was found that even minimal alcohol dosage can result in significant cognitive damages. Half of the adult ADHD demographic also presents addictive tendencies. [9]

\subsection{Maternal pregnancy psychological struggles}

As discussed, pregnancy habits play a huge role in the development of the child. Now research begins to study the link between maternal mental health struggles before and during pregnancy and offspring attention deficit hyperactivity symptoms. A wide study in Italy suggested that children of mothers who had experienced depression, anxiety and sleep disorders when pregnant had a higher ADHD count than those of mothers who have never experienced such struggles. When the disorders were faced during pregnancy the ADHD counts were higher than when faced prepregnancy. Another study in Finland linked steady depressive symptoms during pregnancy with higher ADHD symptoms in children as well. Post-natal depressive symptoms contributed similarly to pre-pregnancy symptoms, having lesser impacts than the manifestations during pregnancy but played a part nonetheless. In this case, prevention plays a very important role and the best option would be the regular psychological tabbing of future mothers. [11] [12] 


\section{Socioeconomic Factors}

A large scale study in Sweden examining the social issues behind children being medically treated for ADHD found very increased odds within children in special social situations regarding the appearance of the disorder. In specific, children being cared for by a single parent were more than $50 \%$ more likely to be on ADHD medication. Women with secondary education had a $130 \%$ chance of their children being treated for the disorder. Families on welfare had a $135 \%$ chance of having their kids on medication. Another angle that the researched demographic gave insight to is the possible role of gender in the frequency of the disorder. Boys were observed to be three times more likely to be affected than girls. However, both sexes were equally affected by social factors. [8]

\section{$5 \quad$ Early Electronics Use}

The use of electronics has become extremely widespread in recent times. Childhood is a very suggestive age for electronics to have an impact on a person's brain development. Not only is excessive electronic use generally unhealthy, but it is also linked with ADHD symptoms during childhood. The use of computers and other media between children was calculated to an average of 3 hours per day. (With the passing of time and thus the mass spreading of new technological abilities with increased accessibility, this calculation may have augmented and may augment more in the future). Also, many cases of internet addiction have developed. Studies confirm that more electronic usage equates to more severe ADHD symptoms. The domain of games is especially perilous to children with ADHD. They are deliberately designed in small parts that don't require much attention, giving encouraging rewards to keep the interest going. This leads children to keep wanting to play for extended periods and creates interest to keep returning to the game. [10]

\section{Parenting Style}

The way children diagnosed with the disorder are handled through parenting, can be an extreme step towards positive change or a very big burden to the existing symptoms. Even if parents have the child's best interests in mind, unhelpful parenting methods can be unknowingly adopted without the right consultation. In fact, research questioning mothers of children with ADHD gave a paramount insight into how the parent-child relationship is affected. According to the results of the research, parenting style was heavily influenced by five factors: the intensity of child disruptive behavior, higher child age, having an only child, augmented social isolation and the type of parental sense of efficiency. Those are factors that tend to push towards incompetent parenting methods. The amount of influence that these factors possess can lead up to $32 \%$ when combined. Ineffective parenting methods are proven to be a big obstacle for a child with ADHD. Hence, family consultation is an important step in families 
with children with the disorder as it provides the advantage of a specialist keeping tabs on parenting style and child behavior. Another necessary step is parents being informed about the disorder and ways of dealing with the symptoms. [13]

\section{Drawbacks of ADHD Medication}

ADHD, more often than not, is accompanied by further emotional or behavioral disorders (75\% - 80\% of cases). ADHD medication has been found to aggravate emotional disorders (or cause them when previously nonexistent). [14] This information is discouraging especially considering the mass numbers of children and adults on medication. Half of the children diagnosed with the disorder are on medication and in most cases, medication continues into adulthood. Many of the patients on ADHD drugs resort to taking multiple medications to treat emotional disorders as well. The majority of the supplementary medication lacks research and is not officially approved. Additionally, there are long term effects of ADHD medications that are not fully documented or studied. Nevertheless, there have been considerable cases where those drugs were linked with increased risk of cardiac malfunction, sudden death, liver damage and psychotic events. [15] In 2008, official instructions (NICE) were announced stating that ADHD medication should be used as a last option, for very severe and untreatable symptoms. Warning labels were also added to ADHD medicine informing about the potential side-effects.

\section{Alternative ADHD Treatments}

\subsection{Brain training}

One key brain function closely connected with ADHD is working memory. The appearance of the disorder is generally accompanied with poor working memory. Students with ADHD were found $400 \%$ more likely to have a deficient working memory. There is plenty of research to support that targeted brain training on working memory can not only improve working memory itself but intelligence and attention as well. [16] Improvements in working memory were proven to be linked with reduced hyperactivity, inattentiveness, and impulsivity in patients suffering from ADHD. Furthermore, patients experienced an improvement in their concentration and attention span, following the working memory training. [17]

Further research used neurofeedback to monitor brain training in patients. This practice uses electrodes to determine activity in different areas of the brain, while using brain training games, targeting specific areas of the brain and acting according to the responses of the patient's brain. In ADHD patients particularly, the emphasis is given on the treatment of areas of the brain and functions affected by the disorder. Brain training using neurofeedback was proven to be of even higher quality than normal computerized brain training. More importantly, neurofeedback patients showed similar or higher progress than patients on medication. [18] 


\subsection{Mindfulness and meditation}

Meditation was proven to be a potent method of fighting ADHD. During one study amongst several adult and teenage patients who took a 2-month mindfulness course, the results were very satisfying. The patients were monitored before the course and after its conclusion. In the end, there were major improvements in ADHD symptoms and performance. Symptoms of anxiety and depression were also very positively affected. [19]

Another research had children aged 11-14 practice the transcendental meditation technique at school. A meditation exercise took place twice a day in their curriculum guided by teachers who were previously trained by experts on the particular technique. The students were tested and evaluated over for three months. This included an evaluation by teachers and parents, self- evaluation and performance tests. The results were determined after the three-month mark and reported considerable progress. Statistical analysis of the results revealed stress and anxiety levels were notably lower while ADHD symptoms and executive function were drastically improved. [20]

\section{Conclusion}

Knowledge of early causes can be paramount in ensuring prevention for as many of them as possible. Those that are not possible to control (e.g. premature birth) are also important to be of knowledge and can lead to the ability to effectively providing help. Getting information on the disorder is a fundamental step in helping affected children. More importantly, care and acceptance on the parental side are what will set the foundation for a strong connection and a steady road to betterment. Anxiety and discouragement can only drift a parent-child relationship apart. Despite the popularity of ADHD medication and the "quick solution" they may provide, their selection requires a lot of thorough examination. The drawbacks are a big factor to consider as well as the fact that dependency on them can form and carry into adulthood. Keeping in mind that psychological trauma may lie behind intense symptoms is important. Concluding we can underline that early intervention could lead to significant improvements of the children with ADHD.

\section{References}

[1] 3 Areas of Executive Function. The Understood Team. www.understood.org

[2] Understanding ADHD. The Understood Team. www.understood.org

[3] Attention Deficit Hyperactivity Disorder: Causes of ADHD. WebMD Medical Reference Reviewed by Smitha Bhandari, MD. www.webmd.com

[4] S.-N. James, A.-S. Rommel, C. Cheung et al. Association of preterm birth with ADHDlike cognitive impairments and additional subtle impairments in attention and arousal malleability. Psychol Med. 2018 Jul; 48(9): 1484-1493. https://doi.org/10.10 17/s0033291717002963 
[5] Franz AP, Bolat GU, Bolat H et al. Attention-Deficit/Hyperactivity Disorder and Very Preterm/Very Low Birth Weight: A Meta-analysis. Pediatrics. 2018 Jan;141. https://doi.org/10.1542/peds.2017-1645

[6] Langley K, Rice F, van den Bree MB, Thapar A. Maternal smoking during pregnancy as an environmental risk factor for attention deficit hyperactivity disorder behaviour. A review. Minerva Pediatrica [01 Dec 2005, 57(6):359-371].

[7] Kristin Gustavson, PhD, Eivind Ystrom, PhD, Camilla Stoltenberg, MD, PhD et al. Smoking in Pregnancy and Child ADHD. Pediatrics. 2017 Feb; 139(2): e20162509.doi: 10.1542/peds.2016-2509.

[8] A Hjern GR Weitoft F Lindblad. Social adversity predicts ADHD-medication in school children - a national cohort study. ACTA Paediatrica Volume 99, Issue 6, June 2010, Pages 920-924. https://doi.org/10.1111/j.1651-2227.2009.01638.x

[9] Burger PH , Goecke TW , Fasching PA, Moll G et al.How does maternal alcohol consumption during pregnancy affect the development of attention deficit/hyperactivity syndrome in the child. Fortschritte der Neurologie-Psychiatrie [07 Jul 2011, 79(9):500-506].

[10] Margaret D. Weiss, Susan Baer, Blake A. Allan, Kelly Saran, Heidi Schibuk. The screens culture: impact on ADHD. ADHD Attention Deficit and Hyperactivity Disorders December 2011, Volume 3, Issue 4, pp 327-334. https://doi.org/10.1007/s12402-011-0065-Z

[11] Vizzini L, Popovic M, Zugna D, Vitiello B, Trevisan M, Pizzi C, Rusconi F, Gagliardi L, Merletti F, Richiardi L..Maternal anxiety, depression and sleep disorders before and during pregnancy, and preschool ADHD symptoms in the NINFEA birth cohort study. Epidemiol Psychiatr Sci. 2018 Apr 18:1-11 https://doi.org/10.1017/s2045796018000185

[12] Elina Wolford et al.Maternal depressive symptoms during and after pregnancy are associated with attention-deficit/hyperactivity disorder symptoms in their 3- to 6-year-old children. PLoS One. 2017; 12(12): e0190248. https://doi.org/10.1371/journal.pone.0190248

[13] Deirdre P. McLaughlin, Christine A. Harrison. Parenting Practices of Mothers of Children with ADHD: The Role of Maternal and Child Factors. Child and Adolescent Mental Health Volume 11, Issue 2 May 2006, Pages 82-88. https://doi.org/10.1111/j.14753588.2005.00382.x

[14] William Copeland, Lilly Shanahan, E. Jane Costello, Adrian Angold. Configurations of common childhood psychosocial risk factors. The Journal of Child Psychology and Psychiatry. Volume50, Issue 4, April 2009, Pages 451-459. https://doi.org/10.1111/j.14697610.2008.02005.x

[15] Centers for Disease Control and Prevention (CDC).Mental health in the United States. Prevalence of diagnosis and medication treatment for attention-deficit/hyperactivity disorder--United States, 2003. https://doi.org/10.1001/jama.294.18.2293-b

[16] Tracy Alloway, PhD. ADHD and Working Memory. tracyalloway.com

[17] Torkel Klingberg et al. Computerized Training of Working Memory in Children With ADHD-A Randomized, Controlled Trial. Child and Adolescent Psychology Volume 44, Issue 2, February 2005, Pages 177-186. https://doi.org/10.1097/00004583-200502000$\underline{00010}$

[18] Holger Gevensleben, Birgit Holl, Björn Albrecht. Neurofeedback training in children with ADHD: 6-month follow-up of a randomised controlled trial. European Child \& Adolescent Psychiatry, September 2010, Volume 19, Issue 9, pp 715-724. https://doi.org/10.10 07/s00787-010-0109-5

[19] Lidia Zylowska, Deborah L Ackerman, May H Yang et al. Mindfulness Meditation Training in Adults and Adolescents With ADHD: A Feasibility Study. Journal of Attention Disorders, Volume: 11 issue: 6, page(s): 737-746. https://doi.org/10.1177/1087054707308502 
[20] Sarina J. Grosswald et al. Use of the Transcendental Meditation Technique to Reduce Symptoms of Attention Deficit Hyperactivity Disorder (ADHD) by Reducing Stress and Anxiety: An Exploratory Study. Current Issues in Education, Volume 10, Number2December, 2008ISSN 1099-839X.

\section{Authors}

Anna Maria Driga, is with N.C.S.R. "Demokritos", Institute of Informatics and Telecommunications, Net Media Lab \& Brain-Mind R\&D, Agia Paraskevi, 153 10, Athens, Greece (e-mail: anna.maria.driga@gmail.com).

A. Drigas is with N.C.S.R. "Demokritos", Institute of Informatics and Telecommunications, Net Media Lab\& Brain-Mind R\&D, Agia Paraskevi, 153 10, Athens, Greece (e-mail: dr@iit.demokritos.gr).

Article submitted 2019-07-05. Resubmitted 2019-08-19. Final acceptance 2019-08-10. Final version published as submitted by the authors. 